\title{
Des souches locales aux ferments lactiques spécifiques: démarche de recherche-action sur l'activation et la gestion d'une ressource biotechnique
}

\author{
Erick Casalta ${ }^{1}$ \\ Pascale Bona ${ }^{2}$ \\ 1 Inra UMR SPO \\ bâtiment 28 \\ 2 , place Viala \\ 34060 Montpellier cedex 01 \\ France \\ <casalta@supagro.inra.fr> \\ 2 GIE Levitu per Casgi Corsi \\ 20270 Aleria \\ France
}

\begin{abstract}
Résumé
Cette étude présente la démarche de recherche-action entreprise en Corse, avec un groupe de fromagers, pour développer des ferments lactiques spécifiques. Basée sur la participation directe des fromagers, la recherche a consisté à concevoir les ferments à partir de souches de bactéries lactiques isolées dans les laits et fromages des producteurs, puis à tester ces ferments dans les fromageries des partenaires. La démarche a permis de passer d'une ressource utilisée de façon individuelle et empirique, les souches locales, à une ressource partagée et gérée collectivement, les ferments spécifiques. Le caractère patrimonial de la ressource et la participation directe des fromagers ont été déterminants dans l'engagement des producteurs dans la mise en place d'une gestion collective de l'outil. Les ferments sont aujourd'hui utilisés quotidiennement. Ils représentent une solution concrète pour s'adapter aux nouvelles conditions de fabrication dans les ateliers fromagers, caractérisées par un appauvrissement des laits en bactéries lactiques.
\end{abstract}

Mots clés : bactérie lactique ; Corse ; fromage ; gestion collective ; label de qualité.

Thèmes : productions animales ; technologies agro-alimentaires ; transformation, commercialisation.

\section{Abstract \\ From local strains to specific starters: the process structuring a research program on the activation and management of a biotechnological resource}

This study presents a research-action program carried out in Corsica with a group of cheese makers to develop specific starters. Based on the direct participation of the cheese makers, this study consisted in designing starters with lactic acid bacterial strains isolated from milks and cheeses of this group of cheese makers. This process modified an individually and empirically used resource, local strains, into a shared and collectively managed resource, specific starters. Patrimonial features of these strains and the direct participation of the cheese makers were determining factors in the collective management of this resource. These starters are now used daily. They represent a concrete way of meeting the new conditions present in cheese dairies, characterised by low levels of lactic acid bacteria in milk.

Key words: cheese; collective management; Corsica; lactic acid bacteria; quality labels.

Subject: animal productions; agrifood technologies; processing, marketing. omme de nombreuses régions méditerranéennes, la Corse possède une forte tradition pastorale. L'élevage ovin et caprin, de type extensif et orienté vers la production fromagère, $y$ joue un rôle économique et social impor- tant en permettant le maintien d'une population active dans les zones rurales. Contrairement à la Sardaigne, l'activité fromagère s'est peu industrialisée. Le patrimoine fromager corse compte cinq types principaux de fromages fer- 
miers (Calenzana, Niolo, Venaco, Bastelicaccia et Sartenais), des fromages laitiers (pâtes pressées, pâtes molles à croûte fleurie ou lavée) et un fromage de lactosérum, le Brocciu. Excepté ce dernier qui bénéficie d'une appellation d'origine contrôlée (AOC), les fromages de Corse ne sont pas certifiés.

La réglementation européenne relative aux denrées alimentaires, communément appelée "paquet hygiène" (www.agriculture.gouv.fr), impose des exigences en matière d'hygiène et définit des critères microbiologiques, notamment pour les fromages aux laits crus de chèvre et de brebis. Afin de respecter ces critères, les producteurs ont amélioré le niveau d'hygiène lors de la traite et du stockage du lait. Le passage d'un atelier fermier traditionnel à un atelier agréé $\mathrm{CE}$, conçu selon le principe de la marche en avant, modifie les conditions de fabrication. Cette évolution réglementaire et technique peut conduire à l'obtention de laits faiblement chargés en microorganismes, qualifiés de paucimicrobiens. Dans la méthode traditionnelle de fabrication, le fromager emploie uniquement cette flore indigène, de façon empirique, pour acidifier le caillé. Or, dans un lait pauci-microbien, les bactéries lactiques ne sont pas assez nombreuses pour assurer une acidification adaptée et entraîner un égouttage suffisant du caillé. De plus, les micro-organismes présents dans le lait cru contribuent largement, de par leurs activités enzymatiques, au développement des qualités organoleptiques des fromages (Grappin et Beuvier, 1997). La microflore présente dans la fromagerie va également coloniser le fromage et contribuer au développement de ses caractères spécifiques. Ainsi, chaque fromagerie peut être considérée comme une niche écologique (Mocquot, 1986).

\section{Problématique de recherche}

L'évolution des conditions de fabrication pose problème sur le plan technologique et sur le plan de la typicité du fromage. Dans cette situation, deux alternatives se présentaient au fromager fermier en Corse : - continuer à employer la flore indigène ; dans les laits faiblement chargés en bac- téries lactiques, l'acidification est insuffisante, et il y a risque de gonflement des fromages en cas d'une contamination par des coliformes;

- utiliser des ferments industriels ; n'étant pas conçus pour ce type de fromage, ces ferments peuvent banaliser le produit.

La question était comment s'adapter aux nouvelles conditions de fabrication, dans la perspective de conserver la typicité des produits tout en garantissant la qualité sanitaire définie par la réglementation communautaire. La voie choisie a consisté à mobiliser la ressource souches locales de bactéries lactiques pour développer des ferments spécifiques. Dans cette perspective, une collection de souches locales de bactéries lactiques a été constituée en isolant des souches dans différentes fromageries produisant du fromage type Venaco.

Après l'identification des isolats (Casalta, 1992), la caractérisation des souches a permis de connaitre leurs principales propriétés technologiques, notamment leur capacité acidifiante (Casalta et al., 1995).

Ensuite, une enquête a montré que les fromagers se sentaient concernés par les souches locales principalement parce que c'est une ressource issue de leur territoire. La plupart d'entre eux se sont déclarés intéressés par le développement de ferments élaborés avec ces souches (Casalta et al., 1996).

Cet article présente la démarche de recherche engagée pour passer des souches locales, identifiées et caractérisées au sein d'une collection, à des ferments spécifiques utilisables par les fromagers.

\section{Méthodologie}

La méthode de travail a consisté à travailler en partenariat avec un groupe de fromagers de façon à prendre en compte non seulement les critères techniques considérés par les chercheurs et les techniciens de développement, mais aussi ceux des fromagers dans le développement des ferments. Le groupe était constitué de producteurs de fromage Venaco qui, lors de l'enquête, s'étaient déclarés intéressés par les ferments (dix fermiers et deux laitiers). Le travail s'est déroulé en trois étapes, schématisées sur la figure 1.

La première étape consistait à concevoir les ferments :
- en sélectionnant les souches de la collection selon des critères technologiques (capacité acidifiante, activités lipolytique et protéolytique);

- en associant les souches sélectionnées selon la complémentarité de leurs propriétés technologiques.

Lors de la deuxième étape, les ferments ainsi constitués ont été expérimentés dans les fromageries des membres du groupe, le principe étant que pour chaque essai, à partir d'un même lait, un fromage témoin (fromage habituel du producteur avec ferment non spécifique de type industriel) et un ou plusieurs fromages expérimentaux (avec ferment spécifique) étaient fabriqués et affinés. L'expérimentation a été conduite durant plusieurs campagnes.

Lors de la troisième étape, les éléments suivants étaient présentés aux fromagers et discutés en réunion après chaque campagne d'essai :

- l'appréciation par les fromagers de l'action des ferments, basée sur leurs observations (niveau d'égouttage, implantation de la flore de surface, consistance du fromage en fin d'affinage). Les fromagers pouvaient ainsi comparer leur fromage habituel avec les fromages élaborés avec différents ferments spécifiques; - les résultats des analyses bactériologiques: dénombrements et typage génétique (Casalta et al., 2005);

- les résultats des analyses physicochimiques : évolution du $\mathrm{pH}$, degrés de lipolyse et de protéolyse ;

- les résultats de l'évaluation sensorielle : un jury constitué de fromagers membres du groupe de travail effectuait une épreuve d'authenticité, l'objectif étant d'apprécier les caractéristiques des fromages expérimentaux par rapport à celles du Venaco “ idéal ". Un jury de personnes entraînées appartenant au centre inra (Institut national de la recherche agronomique) de Jouy-en-Josas faisait un test triangulaire pour déterminer si les produits fabriqués avec les ferments spécifiques étaient perçus comme différents des témoins (Casalta, 2003).

Sur la base de ces éléments, chaque fromager prenait une décision. S'il acceptait d'utiliser les ferments spécifiques pour fabriquer son fromage, il choisissait le ferment le mieux adapté à sa fabrication. En cas de refus, les raisons de sa décision étaient prises en compte dans la préparation de la prochaine campagne d'essais et un nouveau cycle débutait. 


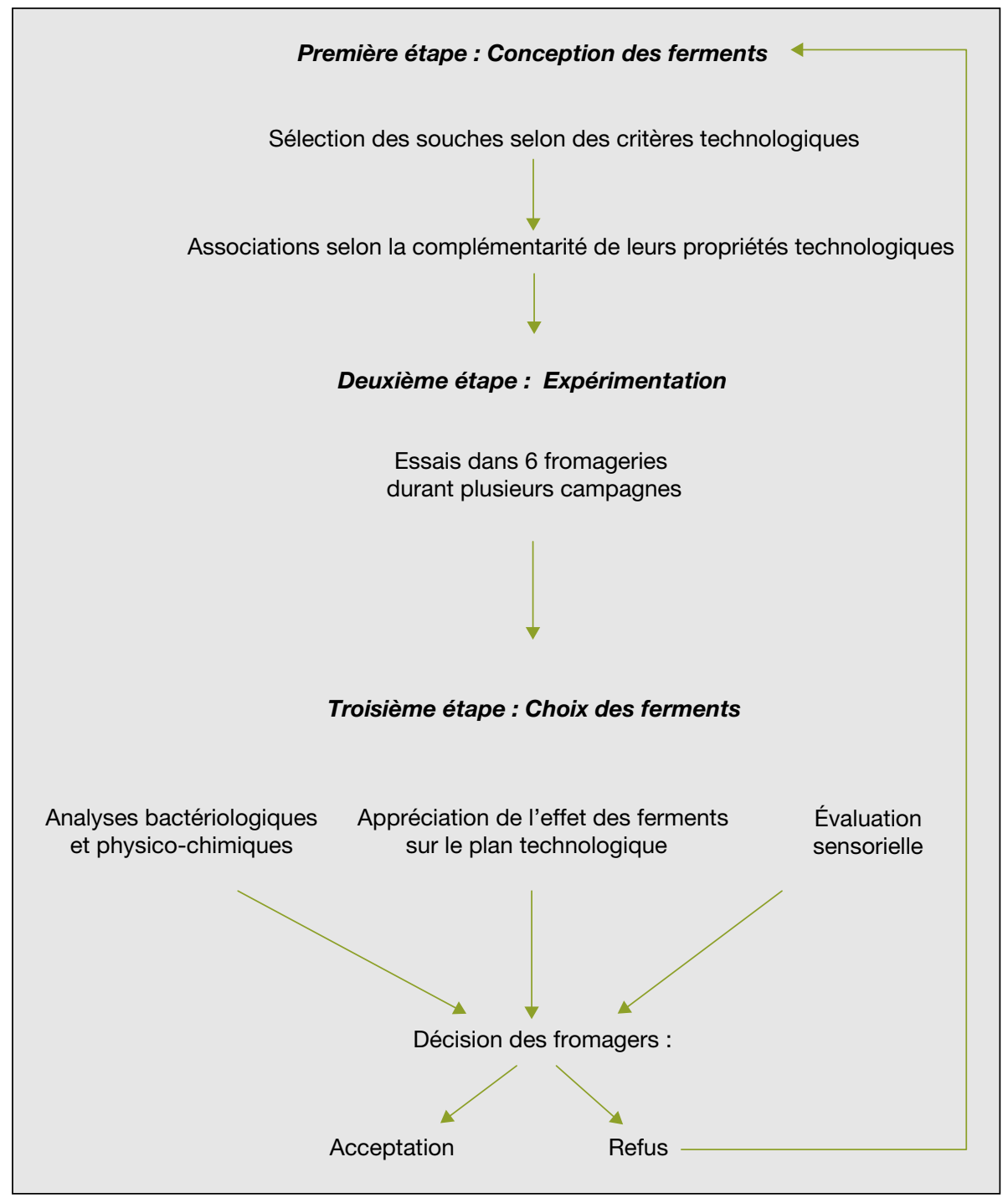

Figure 1. Démarche de développement des ferments

Figure 1. Steps of starters development.

\section{Développement des ferments spécifiques}

\section{Ferments acidifiants et ferments mixtes}

Lors de la première étape, quatre souches acidifiantes et cinq souches d'affinage ont été sélectionnées sur la base de leurs propriétés technologiques.

Deux types de ferments ont ainsi été élaborés :

- les ferments acidifiants composés d'une paire de souches; leur fonction principale est de produire une acidification adaptée au Venaco ; ment. L'implantation de la flore de surface sur les fromages expérimentaux était également jugée satisfaisante. Les fromagers ont aussi observé que les ferments acidifiants donnaient une meilleure consistance que les ferments mixtes en fin d'affinage.

\section{Effet des ferments sur les caractéristiques des fromages mesuré par les analyses et l'évaluation sensorielle}

Le ferment spécifique acidifiant fournissait une acidification moins forte que celle du ferment industriel, dans les premiers jours, ce qui explique l'égouttage moins important observé par les fromagers avec ce ferment. L'introduction d'une souche d'affinage dans le ferment avait pour effet d'augmenter le degré de lipolyse ou de protéolyse, ce qui conduisait à une pâte insuffisamment ferme. Sur le plan sensoriel, les ferments spécifiques acidifiants et mixtes avaient tendance à améliorer les notes globales des fromages obtenues lors du test d'authenticité. De plus, dans la plupart des cas, les fromages fabriqués avec les ferments spécifiques étaient perçus comme significativement différents des fromages témoins au cours du test triangulaire. L'évaluation sensorielle met donc en évidence que l'emploi de ferments spécifiques conduit à des fromages ayant des caractéristiques sensorielles différentes de celles obtenues avec des ferments non spécifiques. Ces différences peuvent s'expliquer par l'effet des souches locales sur les caractéristiques biochimiques du fromage. Le typage génétique ayant démontré que les souches du ferment s'implantent de façon durable dans le fromage, il est probable que cet effet soit provoqué par une action directe de ces souches.

\section{Décision des fromagers}

Sur la base de ces résultats, la majorité des membres du groupe de travail a choisi d'intégrer les ferments spécifiques dans leur fabrication. Selon eux, leur emploi procurait un ou plusieurs des avantages suivants :

- la garantie d'une maitrise des fabrications, les différents ferments testés produisant une acidification suffisante pour inhiber la croissance des coliformes ;

- l'amélioration des caractéristiques sensorielles du fromage ; 
- le renforcement de l'identité du produit: les fromagers pensent que le fait d'incorporer des souches locales dans leur lait renforce les caractéristiques spécifiques du produit. La mise en évidence, par typage génétique, d'un certain équilibre entre les souches du ferment et la microflore indigène a rassuré les fromagers dans le sens où le ferment ne nivelle pas les caractéristiques du produit. L'appréciation de l'action des ferments par le fromager est apparue comme un critère déterminant dans la décision d'accepter ou de refuser l'utilisation des ferments : s'il jugeait l'effet des ferments de façon positive, les autres critères, principalement les résultats de l'évaluation sensorielle, servaient à orienter son choix. Si cet effet était jugé négatif, il décidait de ne pas employer les ferments, sans considérer les autres critères.

Les ferments acidifiants ont été préférés aux ferments mixtes en raison du risque d'aboutir, avec ces derniers, à un produit affiné pas assez consistant. Deux gammes de ferments acidifiants ont ainsi été mises au point : une pour le fromage de brebis et une autre pour le fromage de chèvre.

\section{Organisation de la gestion des ferments}

\section{Création d'un GIE}

Pendant les deux premières campagnes d'essais, la préoccupation principale des fromagers était l'élaboration de ferments adaptés à leur fromage. Cependant, lors des réunions de restitutions annuelles des différences de position sont apparues rapidement. L'objectif des fermiers était l'obtention de la maitrise de la fabrication. Les laitiers étaient motivés par un enjeu technique, le renforcement des caractères typiques du fromage et un enjeu socioéconomique, la volonté de s'approprier les ferments. La confrontation de ces positions a été importante dans la volonté des fromagers de s'engager dans une gestion collective des ferments. En effet, les fermiers seuls se seraient contentés d'être de simples utilisateurs des ferments ; les laitiers seuls auraient probablement tenté de se les approprier dans le but de retrouver une certaine typicité effacée par le fait de pasteuriser le lait.
À partir de la troisième campagne d'essais, les discussions avec le groupe ont principalement porté sur la mise en place d'une gestion des ferments. Le discours des fromagers peut être résumé ainsi : "Les ferments ont été faits avec nos souches, c'est à nous de nous en occuper. "La nature patrimoniale de la ressource a donc été déterminante dans l'appropriation de l'outil par les fromagers et dans leur engagement dans une gestion collective. L'autre élément déterminant a été leur participation directe à l'élaboration de l'outil. Ils se sont sentis impliqués dans la démarche en tant qu'acteurs.

Par ailleurs, le développement des ferments a entraîné la réaction de laitiers industriels et artisanaux qui ont demandé s'il était possible de disposer de ferments locaux pour fabriquer des fromages à partir de lait pasteurisé. Cette réaction a fait émerger la question des conditions d'usage des ferments : qui pourra utiliser les ferments (aire de fabrication), sur quel lait (lait cru ou pasteurisé), pour quel fromage (procédé de fabrication)? Après de nombreuses discussions, le groupe a opté pour un usage des ferments dans la zone d'origine des souches, exclusivement sur du lait cru, pour la fabrication du type traditionnel. Le groupe a choisi de réserver l'emploi des ferments sur du lait cru estimant que les ferments ne devaient pas contrebalancer la perte de typicité entraînée par la pasteurisation.

Souhaitant s'impliquer collectivement dans cette gestion, les fromagers ont créé un groupement d'intérêt économique (GIE) dénommé "Levitu per Casgi Corsi " (levains pour fromages corses). Le groupement a pour objet la mise au point, la production, la commercialisation (à ses membres et à des fromagers non membres) et la protection de ferments spécifiques de fromages de Corse au lait cru, élaborés avec des souches locales de bactéries sélectionnées et cultivées par l'Inra.

\section{Construction du partenariat entre le GIE et I'Inra}

Le partenariat entre le GIE et l'Inra a été officialisé par une licence sur savoir-faire. Inra Transfert, filiale de l'Inra chargée de la valorisation des résultats de recherche a effectué le montage du contrat de licence. Le savoir-faire comprend la sélection et l'association de souches locales de bactéries lactiques ainsi que la conception d'un ensemencement de ces ferments dans les laits. La licence contient également la charte d'utilisation qui définit les conditions d'usage des ferments. Le principe retenu est de limiter l'emploi des ferments à des fromageries situées sur le territoire d'origine des souches et transformant du lait cru de brebis ou de chèvre afin que l'usage de l'outil se fasse dans une perspective de maitrise de la qualité des fabrications et de renforcement de l'identité du produit. Le GIE a en charge la production et la vente des ferments. La préparation et le conditionnement des ferments sont confiés à un organisme tiers, en l'occurrence le Laboratoire d'analyses départementales de Haute-Corse (LDA 2B) implanté à Corte, la prestation de service étant définie par une sous-licence signée entre le GIE et le laboratoire. Ainsi, le partenariat autour de l'outil ferment est organisé en trois pôles (figure 2):

- un pôle professionnel constitué par les fromagers regroupés en GIE chargé de gérer les ferments ;

- un pôle technique constitué par le prestataire de service chargé de la préparation et la Chambre départementale d'agriculture qui apporte son appui technique et logistique par leurs techniciens fromagers ;

- un pôle scientifique constitué par InraTransfert qui s'occupe de la gestion de la licence sur savoir-faire et le Laboratoire de recherches sur le développement de l'élevage (LRDE) qui dispense un appui scientifique et fournit les souches au GIE. La démarche de recherche-action est synthétisée sur la figure 3. La ressource, au départ de la recherche, était constituée par les souches bactériennes, présentes à l'état naturel dans les ateliers fromagers. Utilisée individuellement et empiriquement par les fromagers, c'était une ressource cachée, de par sa nature microscopique, mais aussi parce que les fromagers n'avaient pas vraiment conscience de son rôle dans la qualité du fromage. Le travail de collecte, d'isolement et d'identification a conduit à la constitution de la collection Inra-LRDE. On est passé à une ressource que l'on peut qualifier de publique. La collection INRA-LRDE alimente la Collection nationale de microorganismes d'intérêt laitier dénommée France-MIL (www.francemil.com), représentative de la diversité microbienne des régions fromagères de France et à la disposition de la communauté scientifique. Ensuite, le travail d'expérimentation mené chez les fromagers a permis de développer les ferments qui sont devenus un outil uti- 


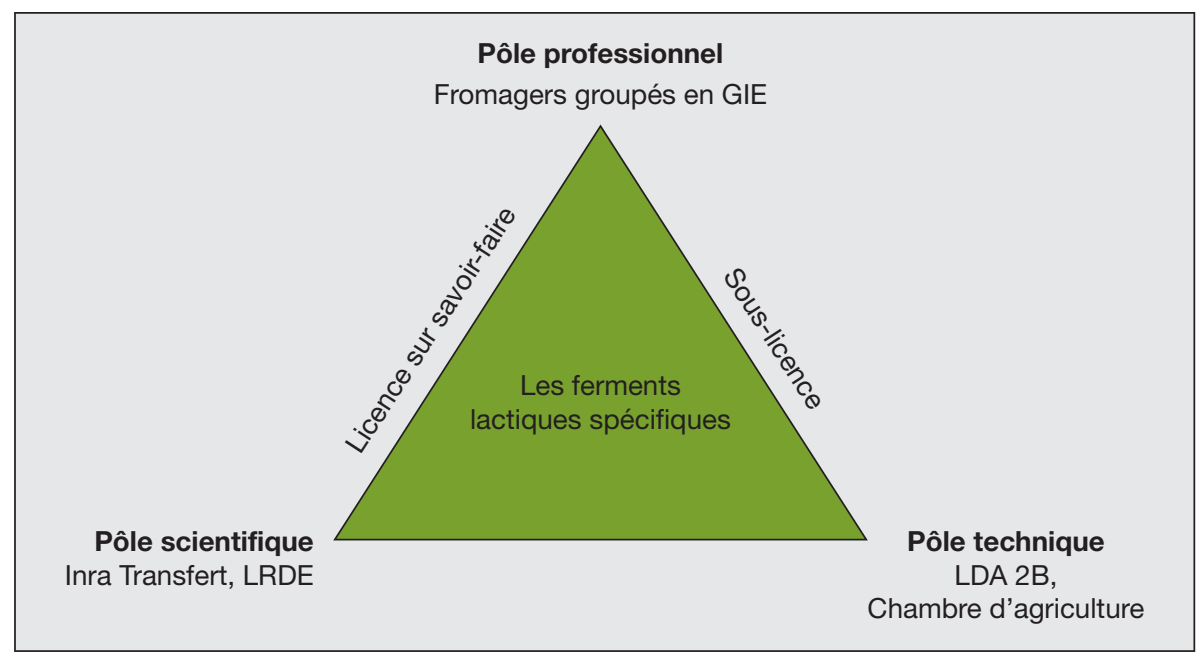

Figure 2. L'organisation du partenariat.

Figure 2. Organization of the partnership.

GIE : groupement d'intérêt économique ; LRDE : laboratoire de recherche sur le développement de l'élevage ; LDA 2B : laboratoire d'analyses départementales de Haute-Corse

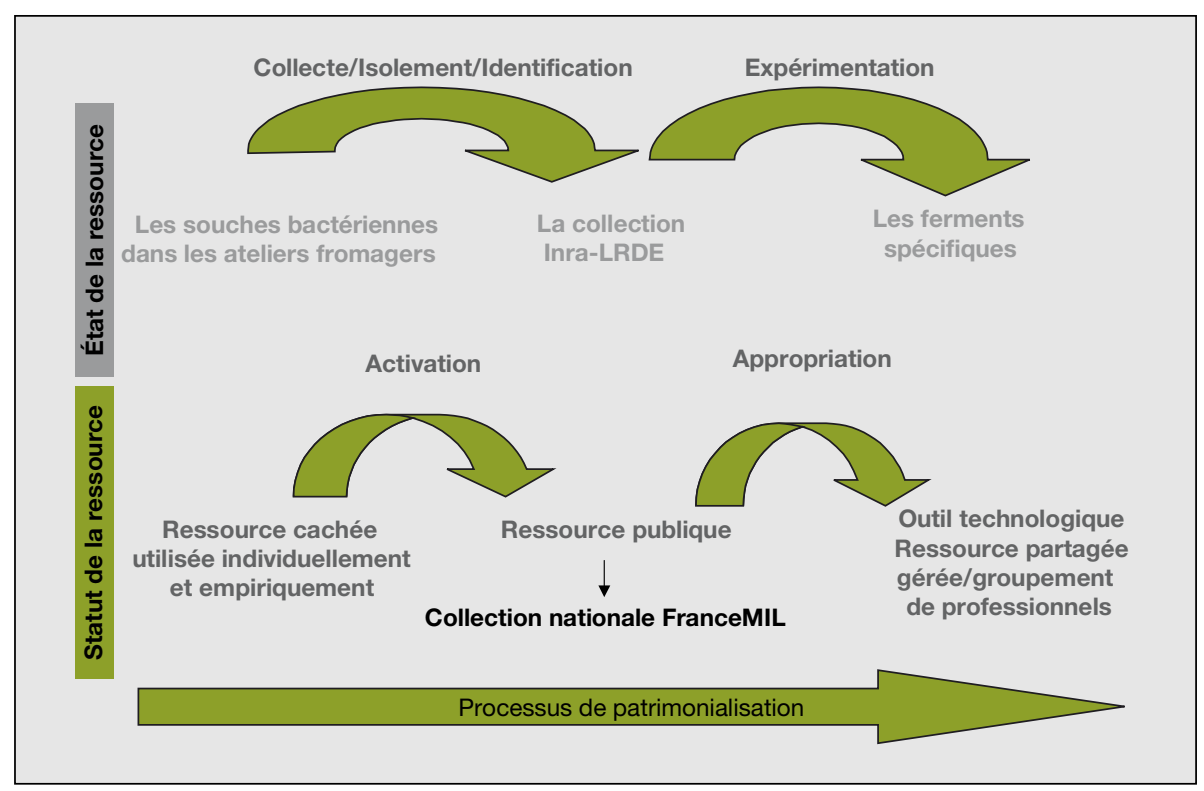

Figure 3. Représentation synthétique de la démarche de recherche-action.

Figure 3. Synthetic representation of the research program process.

LRDE : laboratoire de recherche sur le développement de l'élevage.

lisé selon des critères scientifiques et technologiques. Ils représentent, aujourd'hui, une ressource partagée, gérée par un groupement professionnel et utilisée par une vingtaine de fromagers. Leur emploi n'est pas resté limité aux membres du GIE. D'autres producteurs de Venaco, confrontés à des problèmes d'égouttage ou insatisfaits des ferments industriels, les ont intégrés dans leur fabrication.

\section{Analyse de la démarche}

Généralement, les organismes de recherche mettent au point un outil ou une technique, puis les transfèrent à la profession via des organismes de transfert comme le Centre régional pour l'innovation et le transfert de technologie (CRITT). Les organismes techniques nationaux mènent également des programmes de recherche, notamment sur les filières fromagères traditionnelles, dans lesquels les professionnels sont impliqués. Or, en Corse, le CRITT n'est plus engagé sur les filières animales, et les instituts techniques nationaux ne développent pas de programmes sur la transformation fromagère. Cette situation favorise la mise en place d'un partenariat tel que nous l'avons conçu, entre organismes de recherche, de développement et professionnels. L'originalité de cette démarche réside dans la mutualisation et la mise en synergie des compétences des acteurs: compétences scientifiques, compétences techniques et savoir-faire professionnels. L'avantage de ce processus est que les professionnels sont acteurs à part entière dès le départ et orientent ainsi le développement de l'outil ou de la technique. L'inconvénient réside dans le fait que l'outil ferment a été expérimenté directement chez les producteurs, sans phase préalable en atelier pilote. Cette étape expérimentale, opérée en conditions contrôlées (température, hygrométrie), conduit habituellement à une première série de résultats permettant de comparer l'effet des différents ferments testés et de faire un premier choix de ferments. Notre démarche n'incluant pas cette étape, cette première sélection n'a pu être effectuée. Nous disposons uniquement des données qui proviennent des essais chez les producteurs. Les conditions de fabrication variant selon le producteur, les résultats obtenus dans un atelier ne sont pas forcément reproductibles.

Des ferments fongiques spécifiques ont également été développés en Auvergne pour les fromages AOC de cette région, à la demande de la profession, dans l'objectif de maintenir la typicité et d'améliorer la qualité des produits. La station de recherches fromagères de l'Inra d'Aurillac a sélectionné des souches de moisissures et de levures selon leur capacité à produire le croûtage spécifique des fromages (couleur, texture, épaisseur) défini dans les cahiers des charges des AOC. Les souches sélectionnées ont été testées en plusieurs étapes : testage sur micro-fromages en laboratoire, puis en atelier pilote, essais chez les fromagers, à une échelle semi-industrielle, puis en utilisation industrielle. À l'issue de chaque étape, les souches les mieux adaptées étaient retenues. Lors de la dernière étape, les fromagers choisissaient les souches pour leur propre fabrication (Pradel, 1987). Un GIE, constitué à l'origine par 12 entrepri- 
ses de la région Auvergne, a été créé en 1983, pour produire et commercialiser les ferments. Depuis 2000, et dans l'objectif de diffuser plus largement les ferments, la production et la commercialisation sont confiées au Laboratoire interprofessionnel de production (LIP) dont le statut est une société à actions simplifiée ${ }^{1}$. Aujourd'hui, à côté des ferments spécifiques pour fromages d'Auvergne, le LIP commercialise des levures et moisissures pour d'autres types de fromages traditionnels ou de marque.

Si l'on compare les deux démarches, les principales différences portent sur :

- le contexte : en Corse la filière est peu industrialisée et peu structurée. Hormis le Brocciu protégé par une AOC, les fromages ne bénéficient pas d'un signe officiel de qualité. En l'absence de cahier des charges définissant les caractéristiques du fromage, un travail préalable de caractérisation du fromage a été nécessaire pour disposer de critères de sélection des souches. En Auvergne, les fromages étant certifiés par une AOC, le choix des souches a pu s'appuyer sur les critères de croûtage définis dans les cahiers des charges des AOC.

- la demande: en Corse, elle provient d'un groupe de fromagers, alors qu'en Auvergne elle est le fait de la profession.

Les deux exemples ont en commun :

- l'implication forte et le rôle moteur des professionnels ;
- l'association entre la recherche et les producteurs.

Cette comparaison montre qu'une telle démarche peut être conduite dans des contextes très différents (filière peu structurée et peu industrialisée ou fortement structurée et industrialisée), le dénominateur commun étant la volonté des professionnels de maintenir le lien entre le fromage et son terroir.

\section{Conclusion}

La démarche de recherche-action entreprise a permis d'élaborer, à partir d'une ressource biotechnique à caractère patrimonial, un outil technique permettant aux fromagers de s'adapter à l'appauvrissement de la microflore indigène des laits. Le fait que les fromagers utilisent aujourd'hui ces ferments démontre qu'ils représentent une solution pertinente pour améliorer les caractéristiques sensorielles du fromage. Au cours de ce type de démarche, les professionnels peuvent avoir des points de vue différents sur l'appropriation de la ressource. La difficulté réside dans la construction d'une stratégie commune tenant compte de ces divergences. Cette approche pourrait s'appliquer à l'activation de ressources biotechniques dans d'autres filières, dans lesquelles les producteurs souhaitent garder un lien fort entre le terroir et leur produit.

\section{Références}

Casalta E. Mise au point de levains mésophiles destinés à la fabrication fromagère en Corse. Identification et sélection de souches locales de bactéries lactiques. Mémoire de DEA. École nationale supérieure de biologie appliquée à la nutrition et à l'alimentation (Ensbana), Dijon, 1992.

Casalta E. Bases scientifiques de la qualité du Venaco, fromage traditionnel au lait cru. Mise au point de ferments sélectionnés spécifiques. Thèse de I'université de Bourgogne, 2003, Dijon.

Casalta E, Vassal Y, Desmazeaud MJ, Casabianca F. Comparaison de I'activité acidifiante de souches de Lactococcus lactis isolées de lait et de fromage de Corse. LebensmittelWissenschaft-und-Technologie 1995; 28: 291-9.

Casalta E, Casabianca F, de Sainte Marie C. Des souches microbiennes locales aux levains spécifiques : positions des producteurs de fromages de Corse. Cah Agric 1996 ; 5 : 423-33.

Casalta E, Cachenaut JM, Aubert C, Dufresne F, Noël Y, Beuvier E. Application of specific starters for the manufacture of Venaco cheese. Lait 2005 ; $85: 205-22$.

Grappin R, Beuvier E. Possible implications of milk pasteurization on the manufacture and sensory quality of ripened cheese. Int Dairy $J$ $1997 ; 7: 751-61$.

Mocquot G. Fromages d'hier et d'aujourd'hui. Cult Tech $1986 ; 16$ : 246-51.

Pradel G. Développement de levains spécifiques pour l'amélioration de la qualité des fromages régionaux d'Auvergne. Actes du colloque biotechnologies et industries laitières, Clermont-Ferrand, 1987.

\footnotetext{
$\overline{{ }^{1} \text { Roueyre, }}$ communication personnelle.
} 